\title{
Identifying grouping and non-grouping behaviours in a droplet stream using signal processing techniques
}

\author{
Visakh Vaikuntanathan*1, Kasra Amini ${ }^{1}$, Bernhard Weigand ${ }^{1}$ \\ ${ }^{1}$ Institute of Aerospace Thermodynamics (ITLR), University of Stuttgart, Stuttgart, Germany \\ *Corresponding author email: visakh.vaikuntanathan@ittr.uni-stuttgart.de
}

\begin{abstract}
A distribution of liquid droplets in space, referred to as a 'spray', is seen in many industrial and natural contexts. The local number density of the droplets in such a spray affects the evaporation process within the spray. A spatially non-uniform number density could indicate localized 'droplet grouping', which is the topic of investigation in this paper. We look at a system of a single stream of mono-disperse droplets to understand their grouping behavior. From a single snapshot of a few droplets in the stream at a sufficiently upstream location, we show that it is possible to predict if the droplets will exhibit one of the following three behaviors at a downstream location: (a) grouped: the droplets come closer, (b) non-grouped, equidistant: the droplets remain equidistant from each other, and (c) non-grouped, diffused: the droplets move away from each other as one moves downstream. Using a dummy image of a single stream segment of four droplets as input, it is seen that the autocorrelation of the image and its derivatives have distinct signatures for these three categories. This enables to swiftly identify the tendency of droplets to group in such simple 'one-dimensional sprays' using just a high-resolution snapshot of the droplets as input. This technique is validated with images acquired at a typical experimental condition. For more complex 3D and unsteady sprays other techniques, such as the ones based on Voronoi diagrams, may have to be adopted.
\end{abstract}

\section{Keywords}

Droplet dynamics; droplet grouping; signal processing; autocorrelation.

\section{Introduction}

Liquid sprays, defined as collections of droplets dispersed in an ambient medium, are relevant in many practical applications. Formation of groups or clusters of droplets in such sprays could affect many transport processes (for example, the vaporization of spray droplets [1]). Hence an understanding of how these groups of droplets are formed is essential. A few experimental studies on clustering of droplets in sprays have been reported in the literature [2,3]. These studies deal with identifying the regions in the spray where droplet clusters are observed; however, the mechanisms by which these groups/clusters are formed is not yet clear. One reason for this is the complex nature of the spray itself, which consists of droplets of different sizes and shapes, moving with three-dimensional velocities which vary both in space and time.

In order to understand the fundamental physical mechanisms, which contribute to the grouping of droplets, a simplified system comprising a single stream of monodisperse droplets flowing vertically in the direction of gravity was investigated $[4,5]$. These studies involve capturing the images of the droplet stream at different axial locations to reconstruct the entire droplet stream in space. From the reconstructed image (see, for example, Fig. 5 in [5]), the evolution of the droplets in the stream can be visually observed. This then enables a classification of the droplet dynamic behaviour into one of 'equidistant', 'grouped', or 'diffused' cases. Here 'equidistant' refers to the case where the inter-droplet distance remains invariant in space and time, 'grouped' refers to the case where the inter-droplet distance decreases preferentially in 
some regions of space along the droplet stream, and 'diffused' corresponds to the case where the inter-droplet distance increases in the direction of flow. In this study, an attempt is made to simplify this process of classifying the droplet dynamic behaviour using just one image of the droplet stream captured sufficiently upstream. Often such images at upstream locations cannot be used to classify the droplet dynamics just by visual inspection (see, for example, the first image strip of Fig. 5 in [5]). Here we propose a signal processing technique employing autocorrelation of the image to enable droplet dynamics classification.

The techniques of auto- and cross-correlations have been widely employed in fluid mechanics research. For example, in the field of particle image velocimetry (PIV), autocorrelation has been used to estimate tracer particle density in the flow [6]. The technique of auto-correlation provides a method of comparing a signal (here, the one corresponding to the image) with itself, but offset in space (or, any other relevant variable). The autocorrelation function then exhibits signatures which reveal features of the spatial evolution of the signal. In the case of a droplet stream, the spatial and temporal evolutions of the droplets in the stream are inter-related. For example, as one moves down along the droplet stream, the time coordinate corresponding to a particular droplet could be thought of as increasing. This characteristic thus enables deducing droplet dynamic behaviour (in time) using autocorrelation of a single image taken upstream. The working hypothesis of this study is that the signatures seen in autocorrelation function of a single image as well as its derivatives would enable a clear classification of droplet behaviour in a stream into grouping or non-grouping.

Section II provides a brief overview of the experimental details and discusses the image/signal processing methodologies. The image processing technique is first applied to a set of dummy images to demonstrate its working principle in Sec. III. Further, classification of a real experimental image is also discussed in Sec. III. The main findings are summarized in Sec. IV.

\section{Experimental and image processing details}

A. Brief overview of the experimental test rig: The experimental images presented here are obtained using the experimental set-up for investigating droplet grouping. Please refer to a previous study from our research group for details regarding this set-up [5]. Here, a brief overview of the same is provided for convenience. The test rig consists of a droplet generator, a pressurized liquid flow line, and an imaging system. The flow rate from the droplet generator could be controlled and monitored with valves and pressure gauges installed in the flow line. The droplet generator issues a liquid jet which breaks up to produce a single stream of droplets. With the help of an external excitation applied to a piezo-element in the droplet generator, a monodisperse stream of droplets could be produced. The distances between a droplet and its upstream neighbour and its downstream neighbour could be adjusted using a suitable excitation from a double frequency generator, such that these distances are slightly different from each other near the liquid jet breakup. Figure 1(a) shows a typical image of a single stream of monodisperse droplets at a downstream axial location captured using the experimental set-up.

B. Image processing details: A customized image processing code is written in MATLAB. The image processing algorithm starts with the transformation of the recorded image during the measurements, first to a Gray Scale (GS) and then a Black and White (BW) image. The BW image is a planar discrete distribution of ones and zeroes at the location of pixels' centroid. A 2D scanning of the image domain is performed in two interconnected loops through the rows 
and columns of the BW image. The criteria for entering and exiting a droplet while scanning perpendicular to the stream direction is checked. The criteria are to check if the BW value of each pixel has changed relative to the previous one, and if so, in which direction; from a 1 to 0 , or vice versa. These criteria provide two coordinates for line scan perpendicular to the stream: the minimum and the maximum of the x-coordinate corresponding to the entry into and exit from the droplet at a given $y$-coordinate. The difference between the maximum and minimum of the $x$-coordinate when plotted versus the corresponding $y$-coordinate gives a 'signal' equivalent to the 1D stream of droplets. Figure 1(b) shows this equivalent signal for the image shown in Fig. 1(a).

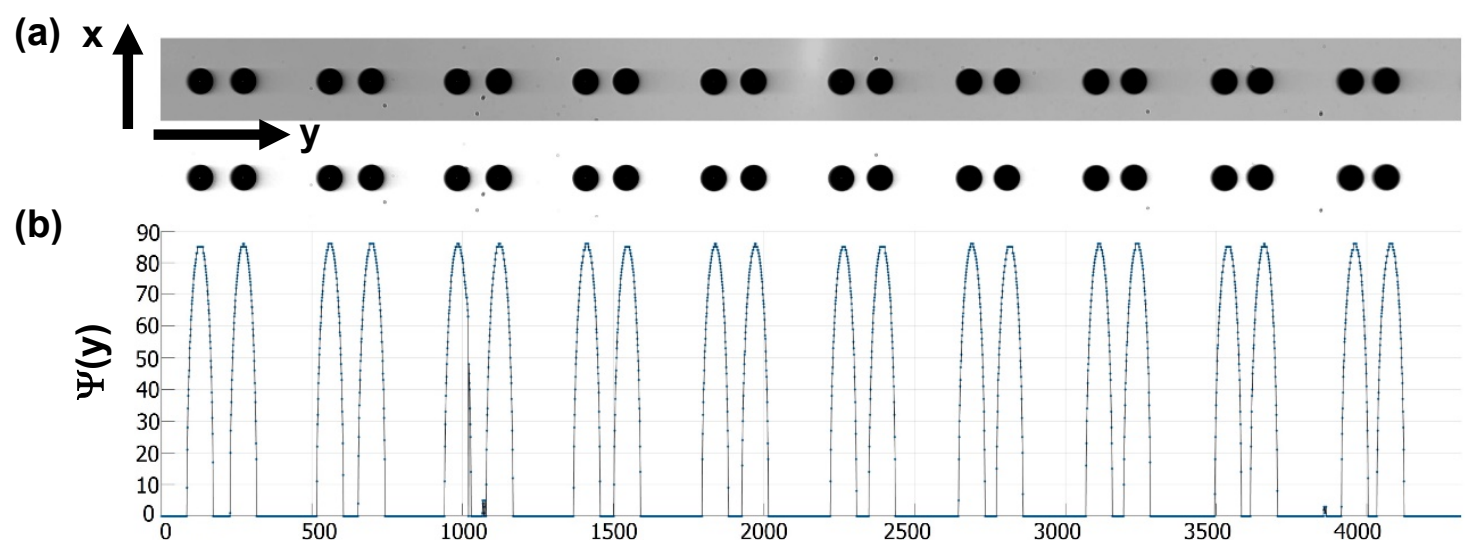

(c)

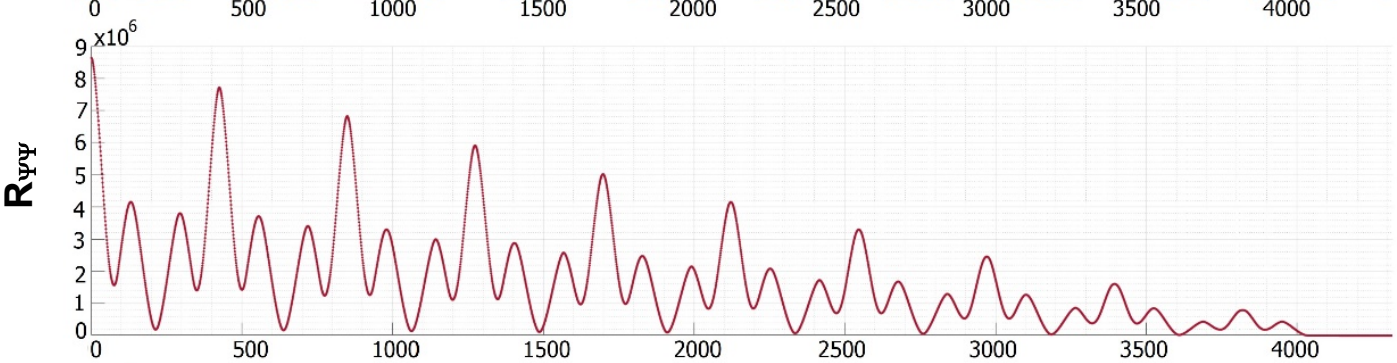

(d)

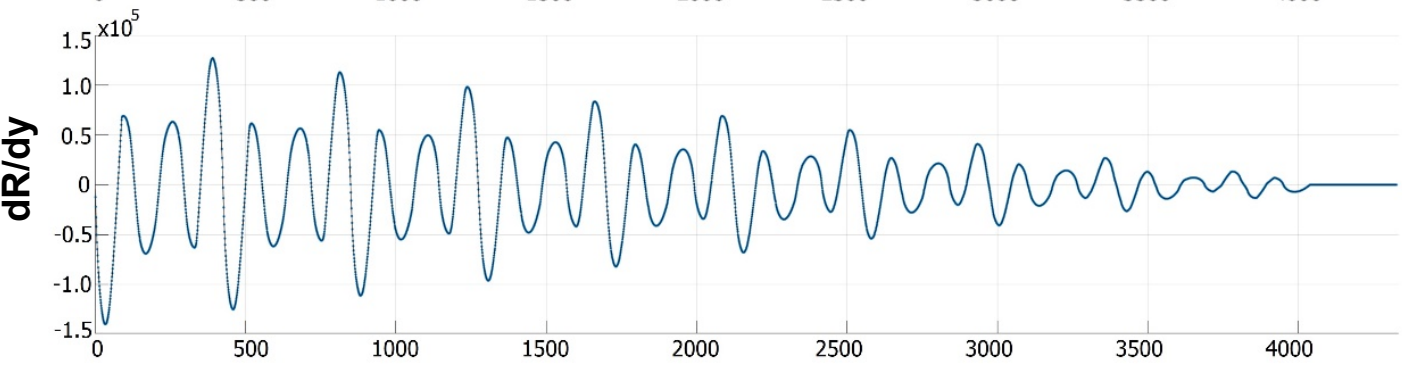

(e)

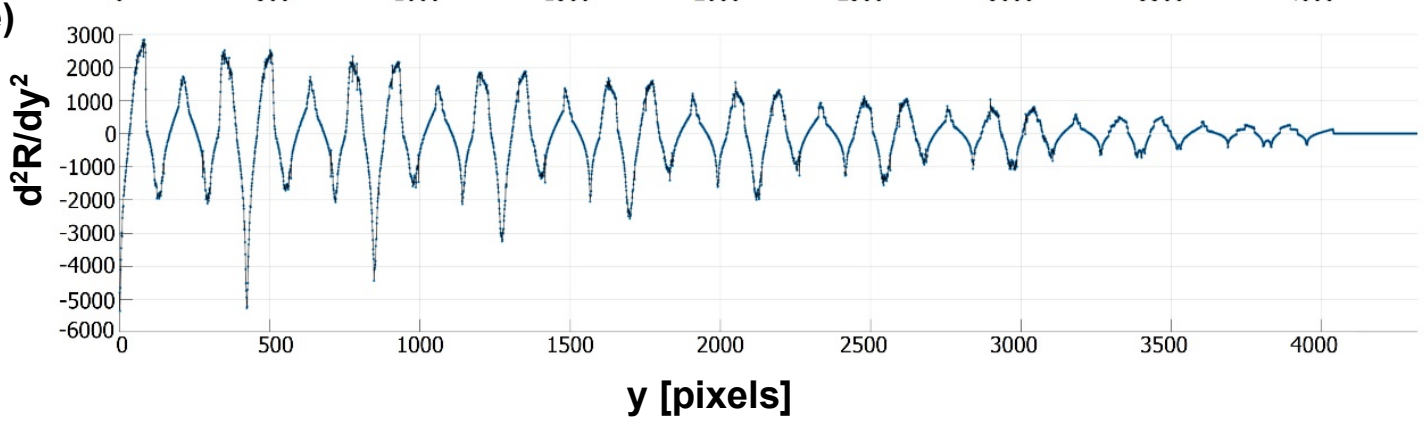

Figure 1. A typical image of a single stream of monodisperse droplets at a downstream axial location is shown in the first row of (a), with the corresponding BW image shown in the second row of (a) (1 pixel $\approx 2 \mu \mathrm{m})$. The droplet motion is along the $y$-direction as shown. The outputs from the image/signal processing algorithm corresponding to the image in (a) are shown: (b) signal, $\Psi(\mathrm{y})$, (c) autocorrelation of the signal, $R_{\Psi \Psi}(\mathrm{y})$, (d) the first derivative of the autocorrelation, $d R_{\Psi \Psi}(y) / d y$, and (e) the second derivative of the autocorrelation, $d^{2} R_{\Psi \Psi}(y) / d y^{2}$. 
Now, the signal can be treated as a mathematical function in space, $\Psi(y)$ to further explore its characteristics. In this paper, the autocorrelation of this signal is explored. As discussed in Sec. I, the techniques of autocorrelation and cross-correlation have been widely employed in signal and image processing in fluid mechanics research. In the present case, moving downstream along the image of the droplet stream (Fig. 1(a)) could be equivalently considered as an advance in time. Hence, a spatial autocorrelation of $\Psi(y)$ would have signatures of the temporal behavior of the droplets in the stream, which may not be immediately clear on visual inspection of the image; for example, whether they come closer together (grouping) or move farther apart (non-grouping). This will enable a classification of their dynamics, without the need for capturing many images downstream. This forms the working hypothesis for this paper. In addition to the autocorrelation of $\Psi(\mathrm{y})$, its first and second derivatives are also calculated to provide more details for the classification of droplet dynamic behavior. The panels (c)-(e) in Fig. 1 show the autocorrelation and its first and second derivatives corresponding to the signal shown in Fig. 1(b).

\section{Results and Discussion}

A. Application of the signal processing technique to classify dummy images: In this section, the signal processing technique is applied to dummy images. Three categories of dummy images are constructed, corresponding to equidistant, grouped and diffused droplets in a stream (see Fig. 2(a)). Figure 2(b) shows the second derivative of the autocorrelation of

(a)
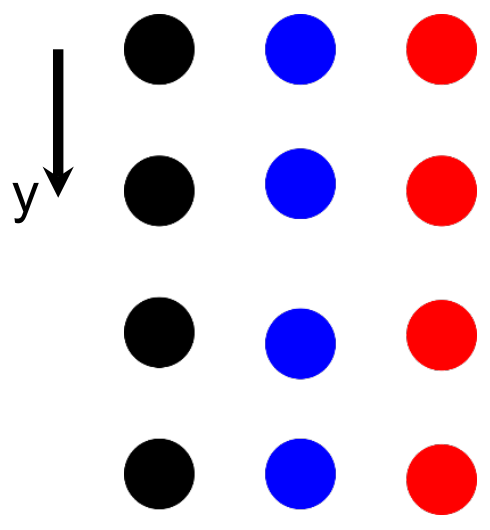

(b)

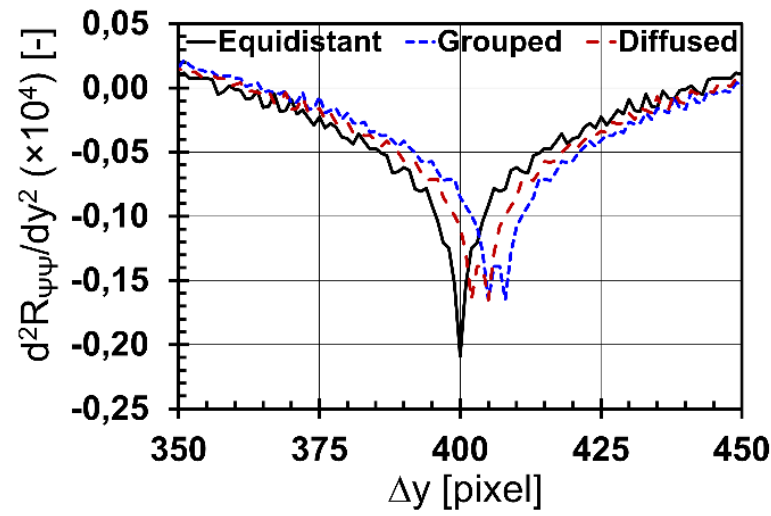

(c)

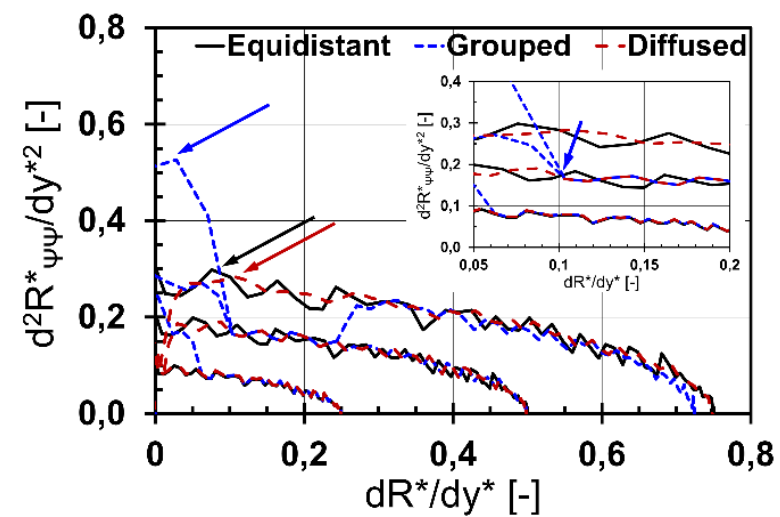

Figure 2. (a) Dummy images of droplet stream segments corresponding to the three typical behaviours of 'equidistant' (left), 'grouped' (middle), and 'diffused' (right). The motion of the droplet stream is considered to be along y. The diameter of the dummy droplet is taken as 100 pixels. (b) Plot showing the variation of the normalized second derivative of autocorrelation $\left(d^{2} R^{*} / d y^{* 2}\right)$, corresponding to the signal from dummy images in (a), with the normalized axial location, $\mathrm{y}^{*}$. (c) Plot showing the variation of the normalized second derivative of the autocorrelation with the normalized first derivative of the autocorrelation corresponding to the signal from dummy images in (a). 
the signals corresponding to these dummy images plotted versus the increment in axial location, $\Delta y$. Only a section of the horizontal axis, which is relevant to differentiate between the three dummy images, is shown here. Qualitatively similar signatures are seen at other values of $\Delta y$. In Fig. 2(b) it is evident that the 'equidistant' category shows only a single downward peak, whereas the 'grouped' and 'diffused' categories show double downward peaks (see at around $\Delta y=400$ pixels in Fig. 2(b)). To distinguish between 'grouped' and 'diffused' categories, an additional criterion is required. For this, the plots showing the trend of the second derivative of the autocorrelation with the first derivative of the autocorrelation are presented in Fig. 2(c). Note that here the values of the first and second derivatives are normalized with their corresponding maximum values and presented as $\mathrm{dR}^{\star} / \mathrm{dy}^{*}$ and $\mathrm{d}^{2} \mathrm{R}^{\star} / \mathrm{dy} \mathrm{y}^{* 2}$, respectively.

It is evident from Fig. 2(c) that there are clear differences between the 'grouped' and 'nongrouped' (that is, 'equidistant' and 'diffused') cases. First of all, the maximum value of the normalized second derivative of the autocorrelation $d^{2} R^{*} / d y^{* 2}$ is greater for the 'grouped' case than for the 'equidistant' and 'diffused' cases (highlighted with arrows in Fig. 2(c)). Secondly, for the 'grouped' case the curve exhibits self-intersection (see at around $\mathrm{dR}^{*} / \mathrm{dy}^{*}=0.1$ shown with an arrow in Fig. 2(c) inset), which is not seen in the other two cases. Hence, with figures similar to Fig. 2(b) and Fig. 2(c), the droplet dynamics could be categorized into one of 'equidistant', 'grouping', or 'diffused'.

B. Application of the signal processing technique to classify a real experiment: Figure 3(a) shows an experimental image, at a sufficiently upstream location of the droplet stream, where it is difficult to visually classify the droplet dynamic behaviour into one of 'equidistant', 'grouped', or 'diffused'. In Fig. 3(b) and Fig. 3(c) the corresponding plots of $d^{2} R^{\star} / d y^{* 2}$ versus $\Delta y^{*}$ and $d^{2} R^{*} / d y^{* 2}$ versus $d^{*} / d y^{*}$ are shown.
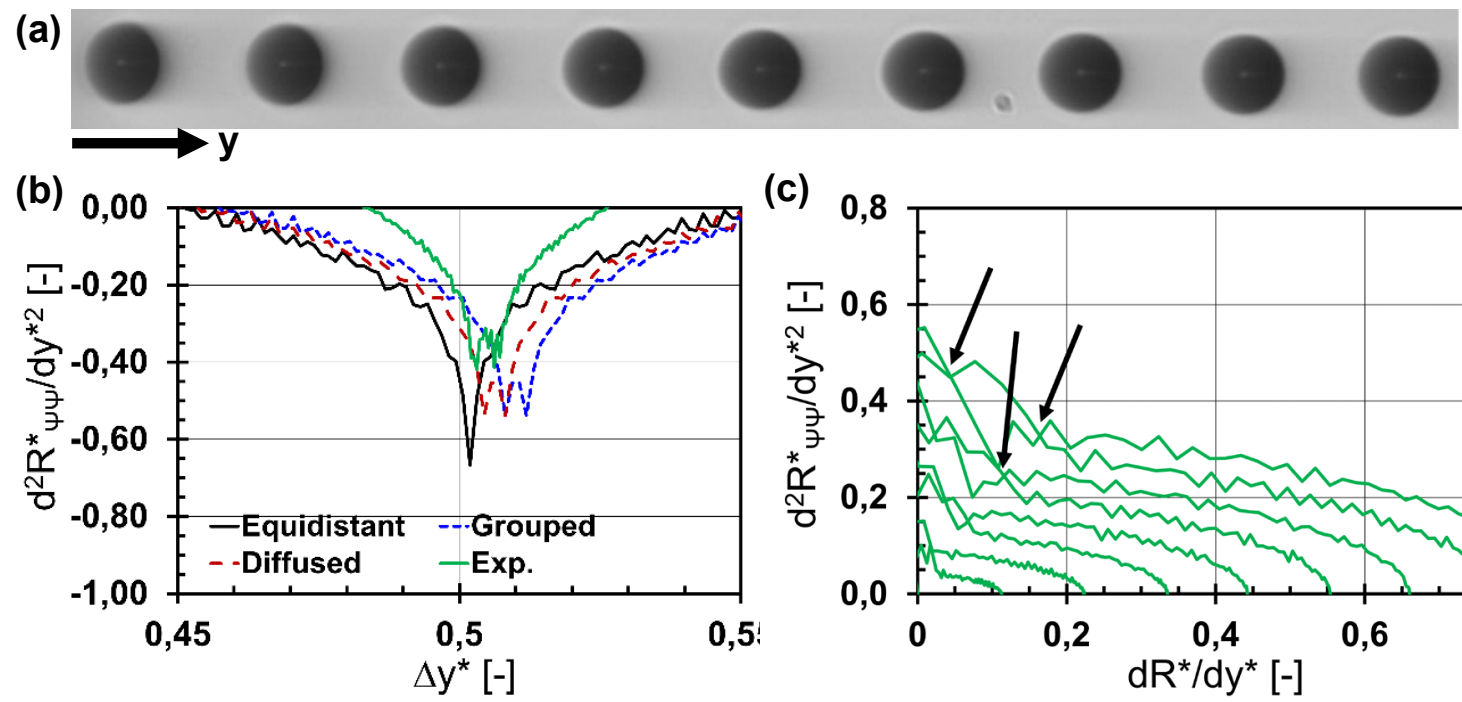

(c)

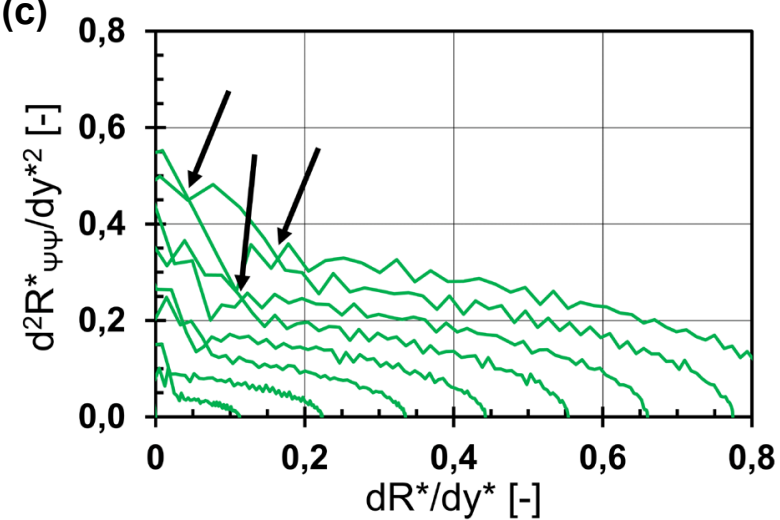

Figure 3. (a) Image of a droplet stream at an axial location close to the liquid jet breakup from experiments. The droplet stream flow is along $y$-direction as shown. Please note that the inclination of the droplet stream seen in this image is corrected in the image processing code. (b) Plot showing the variation of $d^{2} R^{\star} / d y^{* 2}$ with $\Delta y^{*}$ for the experimental image in (a) (green curve). Also shown are the data corresponding to the dummy test cases in Fig. 2(b) replotted in terms of the normalized parameters. (c) Plot showing the variation of $d^{2} R^{\star} / d y^{* 2}$ with $\mathrm{dR}^{*} / \mathrm{dy}^{*}$ for the experimental image in (a), similar to the plots in Fig. 2(c) for the dummy test cases. Some of the coordinates where the curve exhibits self-intersection are shown by arrows. 
It is evident from Fig. 3(b) that the experimental plot also shows a double downward peak, similar to the dummy cases of 'grouped' and 'diffused'. Hence it can be unambiguously stated that the droplet dynamics belongs to one of these categories. In Fig. 3(c), the arrows point to the coordinates where the curve exhibits self-intersection, as seen in the case of 'grouped' in Fig. 2(c). Combining these two observations, it can be predicted that the droplet stream image shown in Fig. 3(a) would undergo grouping downstream.

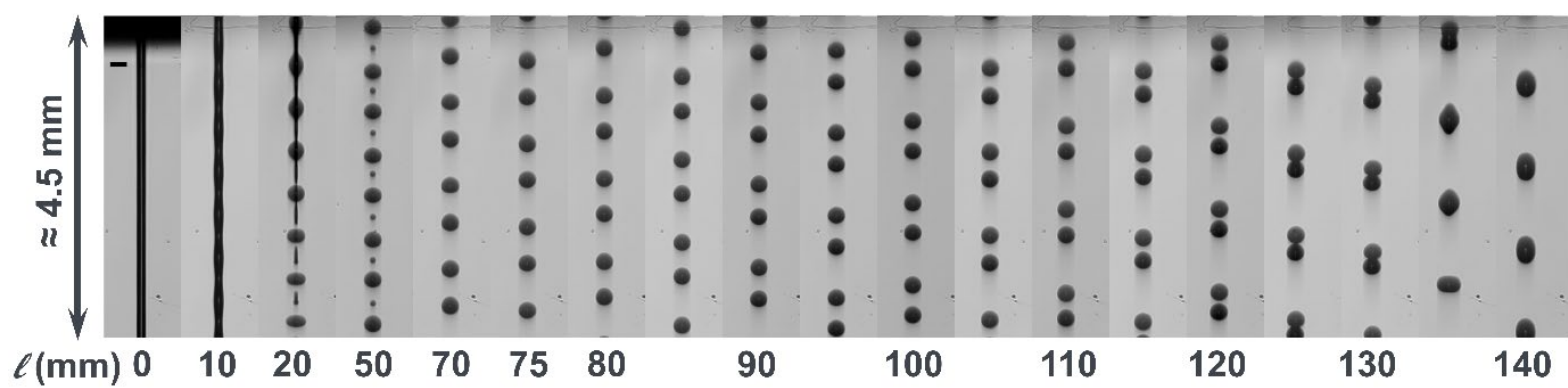

Figure 4. Image sequence showing the complete axial evolution of droplet stream, corresponding to the condition shown in Fig. 3(a). The axial location, $\ell$ of the top of each image strip from the exit of the droplet generator is given at the bottom of the corresponding image strip. Beyond $\ell=80 \mathrm{~mm}$, the top of the consecutive strips are separated by an axial distance of $5 \mathrm{~mm}$. Please note that the axial location of the droplet generator does not coincide with the top of the first image strip. Hence there is a constant negative offset in the values given at the bottom of each image strip. It is evident that the droplets in the stream undergo grouping process as $\ell$ increases from $50 \mathrm{~mm}$ to $135 \mathrm{~mm}$.

Figure 4 presents an image sequence showing the axial evolution of the droplet stream, corresponding to the condition shown in Fig. 3(a). It is clearly seen that the droplets in the stream form pairs, thereby undergoing grouping as the axial location is increased from around $50 \mathrm{~mm}$ to around $135 \mathrm{~mm}$. Hence, this is in line with the prediction from the autocorrelation applied to one image at an upstream location (Fig. 3(a)-(c)). This demonstrates the feasibility of autocorrelation technique to get an initial prediction of the droplet dynamic behaviour in onedimensional droplet streams.

\section{Conclusions}

An image/signal processing algorithm was presented to predict the behaviour of droplets in a single stream of monodisperse droplets. The image captured at a sufficiently upstream location of the droplet stream was used as the input to this algorithm. After converting this raw image into a black and white (binary) image, the image was scanned to identify the peripheries of the droplets in the stream. The width of the droplets was then calculated as a function of the axial location along the stream. This gave a mathematical function, referred to as the 'signal'. Further, this signal was post-processed to obtain autocorrelation of the signal as well as its first and second derivatives. This signal processing method is then applied to dummy images corresponding to three typical cases of 'equidistant', 'grouped', and 'diffused' droplets in a droplet stream. It was shown that the signatures seen in the variations of the second derivative of the autocorrelation with axial location increment and first derivative of the autocorrelation could be used to distinguish between the three droplet dynamic behaviours. This methodology was then applied to a real experimental image of a droplet stream. It was predicted that the droplets would show grouping behaviour, which was consistent with the experimental observation. This technique could, hence, be used for such simple onedimensional sprays (droplet streams). For more complex sprays, other methodologies would have to be explored. 


\section{Acknowledgments}

The authors gratefully acknowledge the financial support from the Deutsche Forschungsgemeinschaft (DFG) through the project 'Investigation of droplet motion and grouping' (Project number 409029509, WE2549/41-1).

\section{References}

[1] Katoshevski, D., Shakked, T., Sazhin, S. S., Crua, C., Heikal, M. R., 2008, Int. J. Heat Fluid Flow, 29, pp. 415-426.

[2] Heinlein, J., and Fritsching, U., 2006, Exp. Fluids, 40, pp. 464-472.

[3] Manish, M., and Sahu, S., 2018, Phys. Fluids, 30, pp. 123305.

[4] Roth, N., Weigand, B., Katoshevski, D., Greenberg, J. B., 2015, Droplet Impact Phenomena and Spray Investigations (DIPSI) Workshop, Bergamo, Italy.

[5] Roth, N., Gomaa, H., Livne, A., Katoshevski, D., Weigand, B., Sep. 6.-8. 2017, 28 ${ }^{\text {th }}$ European Conference on Liquid Atomization and Spray Systems.

[6] Warner, S. O., and Smith, B. L., 2014, Measurement Sci. Tech., 25(6), pp. 065201. 